\title{
Authors' response: it's time to consider integrating the degree of pulmonary fissure completeness into a morbidity risk scoring system for video-assisted thoracoscopic pulmonary resections
}

\author{
Shuangjiang Li, Guowei Che \\ Department of Thoracic Surgery, West China Hospital, Sichuan University, Chengdu 610041, China \\ Correspondence to: Guowei Che. Department of Thoracic Surgery, West China Hospital, Sichuan University, Guoxue Alley No. 37, Chengdu 610041, \\ China. Email: guowei_che@foxmail.com. \\ Provenance: This is an invited article commissioned by the Section Editor Laura Chiara Guglielmetti (Cantonal Hospital Winterthur, Kantonsspital \\ Winterthur, Winterthur, Switzerland). \\ Response to: Lococo F, Nachira D, Margaritora S. Video-assisted thoracoscopic lobectomy in lung cancer patients: a "patient-tailored" surgical \\ approach according to the degree of pulmonary fissure completeness. J Thorac Dis 2018;10:S3092-4. \\ Hishida T. Video-assisted thoracoscopic lung cancer lobectomy for patients with incomplete interlobar fissure: is it a safe and reasonable procedure? \\ J Thorac Dis 2018;10:S3056-7. \\ Lee S, Lee JG. The significance of pulmonary fissure completeness in video-assisted thoracoscopic surgery. J Thorac Dis 2018. [Epub ahead \\ of print].
}

Submitted Oct 05, 2018. Accepted for publication Nov 02, 2018.

doi: $10.21037 /$ jtd.2018.11.13

View this article at: http://dx.doi.org/10.21037/jtd.2018.11.13

We would like to appreciate Dr. Hishida (1), Dr. Lococo with his colleagues (2) and Dr. Lee with his colleagues (3) for their interests and positive comments regarding to our recent single-center retrospective study addressing on the significance of degree of pulmonary fissure completeness (PFC) in video-assisted thoracoscopic surgery (VATS) lobectomy for non-small-cell lung cancer (NSCLC) (4). Meanwhile, we would also like to thank Dr. Laura Chiara Guglielmetti, the Section Editor of the Fournal of Thoracic Disease, for her commission to invite the three professionals in the specialty of thoracic surgery to contribute their great editorials on our work.

There are three take-home messages from our original article (4). All of them are summarized as follows:

(I) Poorly-developed pulmonary fissure is an excellent predictor for postoperative complications after VATS lobectomy. That is the most important finding;

(II) Incomplete degree of PFC is especially relevant to the occurrence of prolonged air leak (PAL, $>5$ days) with its induced pneumothorax and subcutaneous emphysema. In addition, poorly-developed pulmonary fissure is also significantly associated with several major cardiopulmonary complications (CPCs), including the pneumonia, atelectasis and pleural effusion requiring chest tube drainage;

(III) Degree of PFC should be encouraged to help thoracic surgeons inform the surgical patients who are at high morbidity risk. It may also help to select suitable cases in a surgeon's early learning curve or in a teaching program of VATS techniques, in order to avoid a high morbidity rate and train young surgeons more effectively.

Indeed, despite advances in surgical instruments and techniques, poorly-developed pulmonary fissure still poses a great challenge to thoracic surgeons during the intraoperative period since it predisposes to the damage to visceral pleura and an increased probability in experiencing a PAL (5). Nowadays, the VATS approach, as a minimally invasive technique, is sometimes restricted to the detection of minor air leaks and the repair of injured visceral pleura when dealing with the patients with densely fused pulmonary fissures, resulting in the development of a postoperative PAL. In our earlier retrospective cohort study, we have also found that poor degree of PFC is significantly associated with the occurrence of CPCs following VATS 
lobectomy for NSCLC (6). Both of our current two studies provide the initial evidence revealing the significance of degree of PFC for major in-hospital outcomes after VATS lobectomy for NSCLC $(4,6)$.

The thoughtful editorial conducted by Dr. Hishida raises three important questions regarding to our study that merit in-depth consideration (1).

First, Dr. Hishida suggests that it will be informative for us to describe the degree of air leak intraoperatively or just after surgery. In our institution, a PAL was generally originated from an occult air leak that might not be easily detected during the VATS procedure, so we usually paid less attention to record the details of intraoperative air leaks in our operative notes. According to Dr. Hishida's comment, we will take notice of the air leak quantitation in VATS pulmonary resections, especially for the patients with fused inter-lobar fissures.

Second, Dr. Hishida hypothesizes that degree of PFC may also affect the criteria for conversion to thoracotomy due to a dramatically increased surgical difficulty in handling the poorly-developed pulmonary fissures. In our study, we had just excluded the converted cases in advance of the comparative analyses because unexpected conversion could carry confounding influence that might complicate the significance of degree of PFC for morbidity risks. We guess that it may easily lead to an uncontrollable bleeding intraoperatively when trying to dissect the lung parenchyma overlying the major artery within the severely fused interlobar fissures under the thoracoscopic visualization $(5,7)$. That may be a major cause of unexpected conversion, but needs more specific evidence about the impact of degree of $\mathrm{PFC}$ on the conversion rate.

Third, Dr. Hishida wonders the potential differences in postoperative outcomes between VATS procedure and conventional thoracotomy in patients with incomplete pulmonary fissures. In his opinion, we may try to develop a novel technique or just consider a conventional approach for patients with incomplete pulmonary fissures if the quality goal of minimally invasive surgery cannot be achieved with current VATS techniques. We are not sure whether similar results can be observed in conventional thoracotomy or video-assisted mini-thoracotomy because only patients undergoing complete VATS lobectomy were included in our study (4). It needs to be further evaluated in a larger cohort comprising of both VATS and conventional procedures in the future.

On the basis of our findings, we thus recommended that it would be well worth integrating the degree of PFC into a comprehensive risk stratification tool to assess the morbidity risk following VATS pulmonary resections (4). We are very pleased that all three invited professionals also support our opinion and further help optimizing the practice regarding the degree of PFC (1-3).

In the thoughtful editorial conducted by Dr. Lee with his colleagues (3), they suggest a modified fissure assessment model based on the Craig's original classification system (8), the fissure development score-fissure sum average (FDSFSA) system, which has been firstly introduced in their earlier prognosis report (9). In this modified classification system, the pulmonary fissures are divided according to their anatomical regions (i.e., upper major fissure, lower major fissure, minor fissure, and area over the pulmonary artery) and the PFC is divided into four grades (i.e., Grade 0: fully complete fissure; Grade 1: more than 70\% completeness; Grade 2: 30\% to 70\% completeness, and Grade 3: absent to $30 \%$ completeness). And then, the degree of two inter-lobar fissures within a target lobe and the degree of inter-lobar fissure over the pulmonary artery are combined together to calculate the FSA. Finally, an FSA $>1$ will be regarded as the representation of the incomplete pulmonary fissure $(3,4,6,9)$.

It is really an urgent need to reach a consensus on the $\mathrm{PFC}$ definition and standardization. Current evidence indicates that the degree of PFC can be directly assessed by a visual mapping during the surgery, and can also be anticipated by a more accurate analysis of chest computerized tomography (CT) $3 \mathrm{D}$ reconstruction in the preoperative work-up $(2,4,10)$. However, despite encouraging radiological findings, such CT methods still need to be validated, especially for heterogeneous lung diseases, when anticipation of PFC is suboptimal and often under dispute between the radiologists and the surgeons $(10,11)$. PFC evaluation under direct visualization during the surgery may now be more feasible and widely accepted among thoracic surgeons, although this method really depends on the surgeon's area of interest and expertise (12). Therefore, we highly recommend the FDS-FSA scoring system proposed by Dr. Lee with his colleagues as a superior alternative to determine the degree of $\mathrm{PFC}$ in VATS pulmonary resections (9).

Finally, we would like to advocate Dr. Lococo with his colleagues' comment on the practice of PFC indicating that we should better schedule a flexible and "patient-tailored" surgical approach according to the degree of PFC (2). Such a "patient-tailored" model of surgery requires skills in managing a range of surgical techniques (such as the "fissureless" technique, stapler buttressing, energy device 
application and so on) and perioperative care (such as the digital pleural drainage, intensive pulmonary rehabilitation and so on), in addition to a flexibility in using them on a case-by-case basis, thus overcoming strict surgical protocols $(2,5,13-15)$. We think that a "patient-tailored" surgical approach based on the degree of PFC will become an innovative strategy to improve postoperative outcomes in the future. Now, we should investigate how to appropriately integrate the degree of PFC into a comprehensive risk scoring system for VATS pulmonary resections first.

\section{Acknowledgements}

We thank Mrs. Hong Xie, from the Institution of Medical English, West China Medical Center, Sichuan University, Chengdu, China, for her help with the English language editing of this manuscript.

\section{Footnote}

Conflicts of Interest: The authors have no conflicts of interest to declare.

\section{References}

1. Hishida T. Video-assisted thoracoscopic lung cancer lobectomy for patients with incomplete interlobar fissure: is it a safe and reasonable procedure? J Thorac Dis 2018;10:S3056-7.

2. Lococo F, Nachira D, Margaritora S. Video-assisted thoracoscopic lobectomy in lung cancer patients: a "patient-tailored" surgical approach according to the degree of pulmonary fissure completeness. J Thorac Dis 2018;10:S3092-4.

3. Lee S, Lee JG. The significance of pulmonary fissure completeness in video assisted thoracoscopic surgery. J Thorac Dis 2018. [Epub ahead of print].

4. Li S, Wang Z, Zhou K, et al. Effects of degree of pulmonary fissure completeness on major in-hospital outcomes after video-assisted thoracoscopic lung cancer lobectomy: a retrospective-cohort study. Ther Clin Risk Manag 2018;14:461-74.

5. Li SJ, Zhou K, Li YJ, et al. Efficacy of the fissureless technique on decreasing the incidence of prolonged air leak after pulmonary lobectomy: A systematic review and meta-analysis. Int J Surg 2017;42:1-10.

6. Li S, Zhou K, Wang M, et al. Degree of pulmonary fissure completeness can predict postoperative cardiopulmonary complications and length of hospital stay in patients undergoing video-assisted thoracoscopic lobectomy for early-stage lung cancer. Interact Cardiovasc Thorac Surg 2018;26:25-33.

7. Li SJ, Zhou K, Shen C, et al. Body surface area: a novel predictor for conversion to thoracotomy in patients undergoing video-assisted thoracoscopic lung cancer lobectomy. J Thorac Dis 2017;9:2383-96.

8. Craig SR, Walker WS. A proposed anatomical classification of the pulmonary fissures. J R Coll Surg Edinb 1997;42:233-4.

9. Lee S, Lee JG, Lee CY, et al. Pulmonary fissure development is a prognostic factor for patients with resected stage I lung adenocarcinoma. J Surg Oncol 2016;114:848-52.

10. Koenigkam-Santos $M$, de Paula WD, Owsijewitsch $M$, et al. Incomplete pulmonary fissures evaluated by volumetric thin-section CT: semi-quantitative evaluation for small fissure gaps identification, description of prevalence and severity of fissural defects. Eur J Radiol 2013;82:2365-70.

11. Koenigkam-Santos M, Puderbach M, Gompelmann D, et al. Incomplete fissures in severe emphysematous patients evaluated with MDCT: incidence and interobserver agreement among radiologists and pneumologists. Eur J Radiol 2012;81:4161-6.

12. Sedlackova Z, Ctvrtlik F, Miroslav H. Prevalence of incomplete interlobar fissures of the lung. Biomed Pap Med Fac Univ Palacky Olomouc Czech Repub 2016;160:491-4.

13. Li S, Lv W, Zhou K, et al. Does the fissureless technique decrease the incidence of prolonged air leak after pulmonary lobectomy? Interact Cardiovasc Thorac Surg 2017;25:122-4.

14. Lai Y, Su J, Qiu P, et al. Systematic short-term pulmonary rehabilitation before lung cancer lobectomy: a randomized trial. Interact Cardiovasc Thorac Surg 2017;25:476-83.

15. Drahush N, Miller AD, Smith JS, et al. Standardized Approach to Prolonged Air Leak Reduction After Pulmonary Resection. Ann Thorac Surg 2016;101:2097-101.

Cite this article as: $\mathrm{Li} \mathrm{S}$, Che G. Authors' response: it's time to consider integrating the degree of pulmonary fissure completeness into a morbidity risk scoring system for videoassisted thoracoscopic pulmonary resections. J Thorac Dis 2018;10(12):E825-E827. doi: 10.21037/jtd.2018.11.13 